\title{
Clinical efficacy of invigorating qi, nourishing yin, and promoting blood circulation in treatment of type 2 diabetes mellitus.
}

\author{
Xiu-Feng Zhang* \\ Department of Pharmacy, People's Hospital of Rizhao, Rizhao, Shandong, PR China
}

\begin{abstract}
Objective: This study aims to investigate the clinical efficacy of invigorating qi, nourishing yin, and promoting blood circulation in the treatment of type 2 diabetes mellitus.

Methods: Sixty-six patients with type 2 diabetes mellitus admitted to our hospital from February 2015 to January 2017 were recruited in this study. Odd-even method was used to divide the patients into two groups. Patients in the control group $(n=33)$ were given with conventional treatment for type 2 diabetes mellitus, and those in the observation group $(n=33)$ were administered with a conventional scheme involving invigorating qi, nourishing yin, and promoting blood circulation. The TCM syndrome efficacy and the levels of TC, FBG, and FINS were compared between the two groups.

Results: Compared with the control group $(\mathbf{7 2 . 7 3 \%})$, the observation group showed significantly improved TCM syndrome $(96.97 \%)(P<0.05)$ and significantly increased levels of TC, FBG, and FINS (P<0.05).

Conclusion: Treating patients with type 2 diabetes mellitus by invigorating qi, nourishing yin, and promoting blood circulation can ensure TCM syndrome efficacy and increase the levels of TC, FBG, and FINS levels, thereby improving the treatment outcomes.
\end{abstract}

Keywords: Type 2 diabetes mellitus, Invigorating qi, Nourishing yin, Promoting blood circulation, Clinical efficacy. Accepted on October 26, 2017

\section{Introduction}

The number of patients with type 2 diabetes mellitus continuously increases. The patients choose to undergo treatment with oral antidiabetic drug or insulin injection without obtaining the exact glycemic control effect [1]. Moreover, the patients may suffer from hypoglycemia, insulin resistance, weight gain, gastrointestinal adverse reactions, and liver and kidney damage [2]. Thus, developing a rational drug therapy is valuable to patients with type 2 diabetes mellitus [3]. This study aims to explore the value of invigorating qi, nourishing yin, and promoting blood circulation in ensuring clinical treatment efficacy and favorable prognosis for patients with type 2 diabetes mellitus.

\section{Materials and Methods}

\section{General information}

Sixty-six patients with type 2 diabetes mellitus admitted to our hospital from February 2015 to January 2017 were recruited to this study. Odd-even method was used to divide the patients into two groups. The control group $(n=33)$ was composed of 10 males and 23 females aged 41-85 y (average: $54.62 \pm 9.76$ y) and weighing $42-85 \mathrm{~kg}$ (average: $57.43 \pm 6.82 \mathrm{~kg}$ ) and had the disease for 0.6-13.6 y (average: $6.36 \pm 2.13 \mathrm{y}$ ). The observation group $(n=33)$ comprised 11 males and 22 females aged 43-89 y (average: $54.59 \pm 9.79$ y) and weighing $41-85 \mathrm{~kg}$ (average: $57.49 \pm 6.89 \mathrm{~kg}$ ) and had the disease for $0.8-13.9 \mathrm{y}$ (average: $6.39 \pm 2.12 \mathrm{y}$ ). No significant difference in sex, age, weight, and disease duration was noted between the two groups $(\mathrm{P}>0.05)$.

\section{Inclusion and exclusion criteria}

Inclusion criteria: Patients with type 2 diabetes mellitus included in this study were diagnosed on the basis of the following WHO-related diagnostic criteria: Fasting Blood Glucose (FBG) level as low as $7.0 \mathrm{mmol} / \mathrm{L}$ and postprandial blood glucose level as low as $11.1 \mathrm{mmol} / \mathrm{L}$. All the patients were diagnosed with type 2 diabetes mellitus and classified under collateral obstruction by blood stasis on the basis of TCM syndrome. All the patients signed the informed consent.

Exclusion criteria: The following patients were excluded from the study: patients with infection, diabetic ketoacidosis, hyperthyroidism, and gestational diabetes; patients with liver, heart, brain, kidney, and other vital-organ complications or primary disease; women in lactation or pregnancy; patients undergoing long-term insulin treatment or current insulin treatment; patients who showed poor compliance or was unable to cooperate with the researchers; patients allergic to the experimental drug or belonged to allergic constitution; and patients who were unsuitable to participate in this study. 


\section{Method}

Patients in the control group $(n=33)$ underwent conventional treatment for type 2 diabetes mellitus, and those in the observation group $(n=33)$ underwent a conventional scheme that involved invigorating qi, nourishing yin, and promoting blood circulation. Patients in the control group mainly underwent diet control, health education, and exercise therapy as fundamental intervention and were given with $1500 \mathrm{mg} / \mathrm{d}$ metformin. Patients in the observation group were treated by invigorating qi, nourishing yin, and promoting blood circulation. The traditional Chinese medicine administered to the patients at one dose per day consisted of $30 \mathrm{~g}$ of Salvia miltiorrhiza, Astragalus, and Radix pseudostellariae; $12 \mathrm{~g}$ of safflower, moutan bark, and yam; $20 \mathrm{~g}$ of dried rehamnnia root; and $15 \mathrm{~g}$ of Radix ophiopogonis, Schisandra chinensis, Rhubarb, and Polygonatum. Water was added to the medicine until $500 \mathrm{ml}$, and the drug was decocted to $200 \mathrm{ml}$. The patients consumed the herbal decoction warm before meals, twice daily. Treatments for both groups were conducted for 4 months.

\section{Standard criteria}

The treatment outcomes of the patients with type 2 diabetes mellitus were determined on the basis of the standard criteria for TCM syndromes [4]. The scores were as follows: excellent, symptom score decreased to above $2 / 3$; effective: symptom score decreased to $1 / 3-2 / 3$; and invalid, symptom score decreased to no more than $1 / 3$.

\section{Statistical methods}

Data from all the patients were analysed using the statistical software SPSS17.0. The count data (TCM syndrome evaluation results) were presented by (\%) and compared between the groups by using $\chi^{2}$ test. The measurement data
(FINS score) were presented by " $\overline{\mathrm{x}} \pm \mathrm{S}$ " and compared using $\mathrm{t}$ test $[5] . \mathrm{P}<0.05$ indicated statistically significant difference.

\section{Results}

\section{Comparison of TCM syndrome efficacies}

Compared with the control group $(72.73 \%)$, the observation group showed significantly improved treatment efficacy (96.97\%, $\mathrm{P}<0.05$, Table 1).

\section{Comparison of TC levels}

Compared with the control group, the observation group showed significantly improved TC levels $(\mathrm{P}<0.05$, Table 2$)$.

\section{Comparison of FBG levels}

Compared with the control group, the observation group revealed significantly improved FBG levels $(\mathrm{P}<0.05$, Table 3$)$.

\section{Comparison of FINS levels}

Compared with the control group, the observation group demonstrated significantly improved FINS levels $(\mathrm{P}<0.05$, Table 4).

Table 1. Comparison of TCM syndrome efficacies between the two groups of patients with type 2 diabetes mellitus.

\begin{tabular}{llllll}
\hline Group & $\mathbf{n}$ & Excellent & Effective & Invalid & Total effective rate (\%) \\
\hline $\begin{array}{l}\text { Observatio } \\
\mathrm{n}\end{array}$ & 33 & 25 & 7 & 1 & 96.97 \\
\hline Control & 33 & 15 & 9 & 9 & 72.73 \\
\hline $\mathrm{X}^{2}$ & & & & & 7.5429 \\
\hline $\mathrm{P}$ & & & & 0.0060 \\
\hline
\end{tabular}

Table 2. Comparison of TC levels between the two groups of patients with type 2 diabetes mellitus $(\bar{x} \pm S)$.

\begin{tabular}{|c|c|c|c|c|c|c|c|}
\hline Group & Time & $\mathbf{n}$ & $\mathrm{TC}(\mathrm{mmol} / \mathrm{L})$ & TG (mmol/L) & PT (s) & FIB (g/L) & APT (s) \\
\hline \multirow[t]{2}{*}{ Observation } & Before & 33 & $8.11 \pm 0.99$ & $4.70 \pm 0.47$ & $10.95 \pm 0.49$ & $4.31 \pm 0.26$ & $27.97 \pm 3.55$ \\
\hline & After & 33 & $4.77 \pm 0.90$ & $2.93 \pm 0.51$ & $12.51 \pm 0.92$ & $4.39 \pm 0.30$ & $28.60 \pm 3.13$ \\
\hline $\mathrm{t}$ & & & 14.3405 & 14.6608 & 8.5973 & 1.1576 & 0.7647 \\
\hline$P$ & & & $<0.05$ & $<0.05$ & $<0.05$ & $>0.05$ & $>0.05$ \\
\hline \multirow[t]{2}{*}{ Control } & Before & 33 & $7.89 \pm 0.85$ & $4.69 \pm 0.45$ & $10.53 \pm 0.55$ & $3.86 \pm 0.65$ & $27.45 \pm 3.05$ \\
\hline & After & 33 & $5.69 \pm 0.93$ & $4.65 \pm 0.45$ & $11.76 \pm 1.01$ & $4.23 \pm 0.73$ & $32.70 \pm 3.15$ \\
\hline $\mathrm{t}$ & & & 10.0308 & 0.3610 & 6.1439 & 2.1745 & 6.8783 \\
\hline$P$ & & & $<0.05$ & $>0.05$ & $<0.05$ & $>0.05$ & $<0.05$ \\
\hline
\end{tabular}

Table 3. Comparison of FBG levels between the two groups of patients with type 2 diabetes mellitus.

\begin{tabular}{llll}
\hline Group & n & FBg $(\mathrm{mmol} / \mathrm{L})$ & $2 \mathrm{~h} \mathrm{PG} \mathrm{(mmol/L)} \quad \mathrm{HbAlc}(\%)$ \\
\hline
\end{tabular}


Clinical efficacy of invigorating qi, nourishing yin, and promoting blood circulation in treatment of type 2 diabetes mellitus

\begin{tabular}{|c|c|c|c|c|c|c|c|}
\hline & & Before & After & Before & After & Before & After \\
\hline Observation & 33 & $9.01 \pm 0.81$ & $6.62 \pm 0.86$ & $12.09 \pm 1.15$ & $7.95 \pm 1.27$ & $7.52 \pm 0.87$ & $6.09 \pm 0.35$ \\
\hline Control & 33 & $8.73 \pm 0.89$ & $7.67 \pm 0.87$ & $11.81 \pm 0.99$ & $8.66 \pm 1.01$ & $7.35 \pm 0.93$ & $6.56 \pm 0.29$ \\
\hline $\mathrm{t}$ & & 1.3365 & 4.9306 & 1.0599 & 2.5135 & 0.7668 & 5.9400 \\
\hline$P$ & & $>0.05$ & $<0.05$ & $>0.05$ & $<0.05$ & $>0.05$ & $<0.05$ \\
\hline
\end{tabular}

Table 4. Comparison of FINS levels between the two groups of patients with type 2 diabetes mellitus.

\begin{tabular}{|c|c|c|c|c|c|c|c|}
\hline \multirow[t]{2}{*}{ Group } & \multirow[t]{2}{*}{$\mathbf{n}$} & \multicolumn{2}{|l|}{ FINS (mIU/L) } & \multicolumn{2}{|l|}{ ISI } & \multicolumn{2}{|l|}{ BMI (kg/m2) } \\
\hline & & Before & After & Before & After & Before & After \\
\hline Observation & 33 & $19.32 \pm 0.98$ & $15.01 \pm 1.19$ & $-5.55 \pm 0.75$ & $-5.39 \pm 0.51$ & $25.20 \pm 1.45$ & $24.05 \pm 2.29$ \\
\hline Control & 33 & $19.16 \pm 1.01$ & $18.05 \pm 1.42$ & $-5.56 \pm 0.60$ & $-5.92 \pm 0.63$ & $25.55 \pm 1.39$ & $24.26 \pm 1.91$ \\
\hline $\mathrm{t}$ & & 0.6531 & 9.4259 & 0.0598 & 3.7562 & 1.0009 & 0.4045 \\
\hline $\mathrm{P}$ & & $>0.05$ & $<0.05$ & $>0.05$ & $<0.05$ & $>0.05$ & $<0.05$ \\
\hline
\end{tabular}

\section{Discussion}

The rational application of invigorating qi, and nourishing yin, and promoting blood circulation can achieve significant results for patients with type 2 diabetes mellitus [6]. The component Astragalus can tonify the middle-Jiao and Qi and disperse the essence to the lung. Meanwhile, Ophiopogon japonicus, dried Rehmannia root, and Salvia miltiorrhiza help activate and nourish the blood and thus replenish and enhance yin [7]. Schisandra chinensis nourishes and astringes yin and engenders the liquid. Sealwort and Radix pseudostellariae can help nourish the qi to generate fluid and invigorate the spleen and lung [8]. Yam also aids in nourishing the kidney to strengthen yin and tonify spleenic yin. Carthamus tinctorius helps promote blood circulation and remove blood stasis [9]. Rhubarb and Moutan bark can facilitate the clearing of heat, activation of blood, and dispersion of stasis. The compatibility of medicines can ensure the promotion of blood circulation and removal of blood stasis and help supplement qi and nourish yin in patients with type 2 diabetes mellitus [10].

Results revealed that the observation group showed significantly improved TCM syndromes $(96.97 \%)(\mathrm{P}<0.05)$ and significantly increased levels of TC, FBG, and FINS compared with the control group $(72.73 \%)(\mathrm{P}<0.05)$. These findings prove the value of invigorating qi, nourishing yin, and promoting blood circulation in treatment of patients with type 2 diabetes mellitus.

\section{Conclusion}

The rational application of invigorating qi, nourishing yin, and promoting blood circulation can improve the TCM efficacy and increase the levels of TC, FBG, and FINS for clinical treatment of patients with type 2 diabetes. The developed protocol can be used to successfully treat patients with type 2 diabetes mellitus.

\section{References}

1. Gu PY, Yu F, Jin S, Yang Q, Su J, Chen Y, Zhao L, Hu SL. Analysis of serum undercarboxylated osteocalcin level in rats with type 2 diabetes mellitus and the correlation with cognitive impairment. Exp Ther Med 2017; 14: 2603-2607.

2. Shi Y, Chen X, Li Y, Zhang H, Lv Z, Qi Y, Han S. Ursolic acid: is a better hope against liver injury caused by diabetes mellitus? Lat Am J Pharm 2017; 36: 723-729.

3. Zhao LS, Lin YY, Liu Y, Xu CY, Liu Y, Bai WW, Tan XY, $\mathrm{Li} \mathrm{DZ}, \mathrm{Xu}$ JL. Doxazosin attenuates renal matrix remodeling mediated by anti- $\alpha 1$-adrenergic receptor antibody in a rat model of diabetes mellitus. Exp Ther Med 2017; 14: 2543-2553.

4. Chen H, Wu G. Developments in treatments of ulnar coronoid process fracture. Biomed Res India 2017; 28: 3811-3815.

5. Nomani AZ, Nabi S, Ahmed S, Iqbal M, Rajput HM, Rao $\mathrm{S}$. High HbA1c is associated with higher risk of ischaemic stroke in Pakistani population without diabetes. Stroke Vasc Neurol 2016; 1: 133-139.

6. Mou XL, Cao XR, Wang B. Drug-drug interaction between propofol and diabetes treatment drug glimepiride. Lat Am J Pharm 2016; 35: 1673-1677.

7. Ernande L, Audureau E, Jellis CL, Bergerot C, Henegar C, Sawaki D, Czibik G, Volpi C, Canoui-Poitrine F, Thibault H, Ternacle J, Moulin P, Marwick TH, Derumeaux G. Clinical implications of echocardiographic phenotypes of patients with diabetes mellitus. J Am Coll Cardiol 2017; 70: 1704-1716.

8. Jiang J, Zhao J, Yuan Y, Di S. Risk factors associated with acute exacerbation of chronic obstructive pulmonary disease: A retrospective analysis in 4624 patients. Biomed Res India 2017; 28: 3855-3859. 
9. Peters ML, Huisman EL, Schoonen M, Wolffenbuttel BHR. The current total economic burden of diabetes mellitus in the Netherlands. Neth J Med 2017; 75: 281-297.

10. Kamalpour M, Ghalandari H, Nasrollahzadeh J. Short-term supplementation of a moderate carbohydrate diet with psyllium reduces fasting plasma insulin and tumor necrosis factor- $\alpha$ in patients with type 2 diabetes mellitus. J Diet 2017; 28: 1-9.

\section{*Correspondence to}

Xiu-Feng Zhang

Department of Pharmacy

People's Hospital of Rizhao

PR China 